\title{
Utilização de questionários validados para mensurar a adesão ao tratamento da hipertensão arterial: uma revisão integrativa
}

\author{
THE USE OF VALIDATED QUESTIONNAIRES TO MEASURE ADHERENCE TO ARTERIAL \\ HYPERTENSION TREATMENTS: AN INTEGRATIVE REVIEW
}

\section{UTILIZACIÓN DE CUESTIONARIOS VALIDADOS PARA MENSURAR LAADHESIÓN AL TRATAMIENTO DE LA HIPERTENSIÓN ARTERIAL: UNA REVISIÓN INTEGRAL}

\section{José Wicto Pereira Borges', Thereza Maria Magalhães Moreira², Malvina Thaís Pacheco Rodrigues $^{3}$, Célida Juliana de Oliveira ${ }^{4}$}

\section{RESUMO}

Este estudo teve como objetivo analisar na produção científica da saúde coletiva quais questionários validados estão sendo utilizados para avaliar a adesão ao tratamento da hipertensão. Trata-se de uma Revisão Integrativa realizada nas bases de dados SCIELO, MEDLINE e LILACS. Foram selecionados nove estudos que utilizaram sete questionários: Teste de Moriski-Green, Cuestionário de Valoración de Adherencia, Cuestionário MBG, Questionário QAM$-Q$, Teste de Haynes, Escala de Conductas em Salud e Hill-Bone compliance Scale. Concluímos que a avaliação da adesão ao tratamento da hipertensão ainda é campo aberto para pesquisa, os diferentes instrumentos utilizados têm suas limitações e não há um método ideal.

\section{DESCRITORES}

Hipertensão

Terapêutica

Adesão à medicação

Cooperação do paciente

Questionários

\begin{abstract}
The objective of this study was to analyze the scientific production in collective health regarding which validated questionnaires are being used to evaluate adherence to hypertension treatments. This integrative review was performed on the SCIELO, MEDLINE and LILACS databases. Nine studies were selected, which used seven questionnaires: Moriski-Green Test, Cuestionário de Valoración de Adherencia, Cuestionário MBG, Questionário QAM-Q, Haynes Test, Escala de Conductas em Salud, and HillBone compliance Scale. We concluded that the evaluation of hypertension treatment adherence is still an field open of research, due to the fact that the different instruments used have limitations, and there is no ideal method yet identified.
\end{abstract}

\section{DESCRIPTORS \\ Hypertension \\ Therapeutics \\ Medication adherence \\ Patient compliance \\ Questionnaires}

\section{RESUMEN}

Este estudio tuvo como objetivo analizar en la producción científica de salud colectiva cuáles cuestionarios validados están siendo utilizados para evaluar la adhesión al tratamiento de la hipertensión. Revisión integral realizada en las bases de datos SciELO, MEDLINE y LILACS. Fueron seleccionados nueve estudios que utilizaron siete cuestionarios: Test de Moriski-Green, Cuestionario de Valoración de Adherencia, Cuestionario MBG, Cuestionario QAM-Q, Test de Haynes, Escala de Conductas en Salud y Hill-Bone Compliance Scale. Concluimos en que la evaluación de la adhesión al tratamiento de la hipertensión aún es campo abierto para investigación, los diferentes instrumentos utilizados tienen sus limitaciones y no existe un método ideal.

DESCRIPTORES
Hipertensión
Terapéutica
Complimiento de la medicación
Cooperación del paciente
Cuestionarios

${ }^{1}$ Enfermeiro. Especialista em Enfermagem Clínica. Mestrando em Cuidados Clínicos em Saúde da Universidade Estadual do Ceará. Membro do Grupo Epidemiologia, Cuidado em Cronicidades e Enfermagem. Bolsista CAPES. Fortaleza, CE, Brasil. wictoborges@yahoo.com.br 2 Enfermeira. Doutora em Enfermagem pela Universidade Federal do Ceará. Professora do Programa de Pós-Graduação da Universidade Estadual do Ceará. Lider do Grupo Epidemiologia, Cuidado em Cronicidades e Enfermagem. Pesquisadora CNPq. Fortaleza, CE, Brasil. tmoreira@uece.br ${ }^{3}$ Enfermeira. Mestre em Educação. Doutoranda em Saúde Coletiva da Universidade Estadual do Ceará. Professora do Colégio Agrícola da Universidade Federal do Piauí. Membro do Grupo Epidemiologia, Cuidado em Cronicidades e Enfermagem da Universidade Estadual do Ceará. Bolsista CAPES. Fortaleza, CE, Brasil. malvinat@gmail.com ${ }^{4}$ Enfermeira. Mestre em Cuidados Clínicos em Saúde. Doutora em Enfermagem pela Universidade Estadual do Ceará. Professora Adjunta da Universidade Regional do Cariri. Fortaleza, CE, Brasil. celidajuliana@yahoo.com.br 


\section{INTRODUÇÃO}

$\mathrm{Na}$ atualidade, reconhece-se que o genérico impacto das enfermidades sobre as pessoas não pode ser descrito em sua totalidade por medidas objetivas de saúde ${ }^{(1)}$. $O$ desafio de captar objetivamente atributos subjetivos de diferentes temas relacionados ao processo saúde-doença-cuidado vem impulsionando a criação e adaptação de instrumentos, que, uma vez validados, permitem a aproximação com a realidade de modo a subsidiar intervenções mais efetivas ${ }^{(2)}$.

A procura por informações de medidas de propriedades subjetivas motivou muitos pesquisadores a desenvolverem modelos que pudessem estimar essas proprieda$\operatorname{des}^{(3)}$. No âmbito da hipertensão arterial, doença crônica que requer tratamento medicamentoso e não medicamentoso por toda a vida, o grande desafio se faz presente: a adesão à terapêutica como construto subjetivo a ser mensurado.

A adesão ao tratamento da hipertensão é entendida como o grau de coincidência entre o comportamento do usuário e a recomendação do profissional de saúde perante o regime terapêutico(4). Espera-se como resultado o controle da pressão arterial, a redução na incidência ou retardo na ocorrência de complicações e a melhoria da qualidade de vida ${ }^{(5)}$.

Apesar de existirem várias estratégias para avaliar a adesão ao tratamento da hipertensão, não há consenso sobre um padrão ouro(6). Os métodos podem ser diretos, como a dosagem do princípio ativo/ metabólito da droga ou indiretos como a contagem de comprimidos e relato do usuário(7). Alguns autores desenvolveram questionários genéricos para mensurar a adesão ao tratamento em doenças crônicas, também utilizados em pessoas com hipertensão(8).

O uso de instrumentos validados para medir o construto adesão permite uma precisão sobre esse dado subjetivo no cotidiano de cuidado à pessoa com hipertensão. A validade refere-se ao aspecto da medida ser congruente com a propriedade medida dos objetos, demonstrando a representação desse construto na prática ${ }^{(9)}$.

Desse modo, ao utilizarmos instrumentos válidos para medir adesão, estaremos lançando mão de resultados fidedignos que poderão ser utilizados por gestores e equipes de saúde como subsídios no estabelecimento de melhorias nos serviços, culminando com o aumento da adesão. $\mathrm{O}$ objetivo desse estudo foi analisar, na produção científica da saúde coletiva, quais questionários validados estão sendo utilizados para avaliar a adesão ao tratamento da hipertensão.

\section{MÉTODO}

Esta pesquisa foi realizada a partir de uma revisão integrativa da literatura, que tem como base a análise de material, ressaltando a organização e a interpretação no atendimento do objetivo da investigação(10).

A presente revisão integrativa cumpriu criteriosamente seis etapas: seleção de questão norteadora; definição das características das pesquisas primárias da amostra; seleção das pesquisas que compuseram a amostra da revisão; análise dos achados dos artigos incluídos na revisão; interpretação dos resultados e relato da revisão, o que proporcionou o exame crítico dos achados.

Foi realizada a análise dos estudos selecionados, norteada pela pergunta de pesquisa: quais questionários, que passaram por processo de validação, estão sendo utilizados para a avaliação da adesão ao tratamento da hipertensão arterial?

Foi realizada uma pesquisa nas bases de dados eletrônicas SciELO, MEDLINE e LILACS. O período delimitado para a pesquisa dos artigos foi de 2000 a 2009. Foram utilizados os descritores: hypertension, adherence, compliance, test, epidemiologic studies, epidemiology e meansurement, de acordo como a terminologia em saúde DeCS, da Biblioteca Virtual em Saúde. Para sistematizar as buscas foram utilizados os operadores booleanos com o seguinte esquema: (Hypertension and adherence and epidemiology/Hypertension and compliance and epidemiology) nas buscas subsequentes utilizamos esse padrão modificando consecutivamente 0 último descritor, contemplando sistematicamente todos os descritores.

Foram adotados os seguintes critérios de inclusão: artigos que abordassem a adesão ao tratamento da hipertensão arterial analiticamente; disponíveis na íntegra; em língua portuguesa, espanhola ou inglesa publicado entre 2000 e 2009, estudos com dados empíricos de pesquisa. Os critérios de exclusão adotados foram: ser comunicação prévia, revisão de literatura ou revisão teórica.

A fase de coleta de dados ocorreu no período de março a maio de 2010 nas referidas bases de dados eletrônicas. Como resultado da pesquisa identificamos 249 trabalhos potenciais para o estudo, sendo realizadas leituras dos resumos, fazendo-se uma triagem quanto à relevância e à propriedade que responderam ao objetivo do estudo. Após a leitura dos resumos, foram pré-selecionados 33 trabalhos disponibilizados na íntegra, relidos e novamente selecionados. Desses, excluímos: cinco artigos sobre pessoas com hipertensão e outra doença, seis que abordavam adesão sem mensurá-la, três que abordavam a adesão dos profissionais às diretrizes clínicas de manejo da hipertensão e dois artigos teóricos. Desse modo, chegou-se a um número de 17 estudos dentre os quais
Utilização de questionários validados para mensurar a adesão ao tratamento da hipertensão arterial: uma revisão integrativa Borges JWP, Moreira TMM, Rodrigues MTP, Oliveira CJ 
nove utilizavam questionários validados para analisar a adesão ao tratamento da hipertensão que compuseram a amostra da pesquisa.

As variáveis selecionadas para análise foram: tipo de estudo, local do estudo, sujeitos, amostra, ano de realização, coleta de dados, instrumentos de avaliação da adesão ao tratamento, prevalência da adesão ao tratamento.

Para garantir a validade da revisão, os estudos selecionados foram analisados detalhada e criticamente, procurando explicações para os resultados diferentes ou conflitantes $^{(10-11)}$

\section{ANÁLISE E DISCUSSÃO DOS RESULTADOS}

Sobre a caracterização dos estudos, os artigos apresentam diferentes características no que se refere ao país em que foram realizados, aos sujeitos e ao delineamento metodológico. Essas características são evidenciadas no Quadro 1.

Quadro 1 - Caracterização da produção científica da área de saúde coletiva que utilizou questionários validados para avaliar a adesão ao tratamento da hipertensão - Fortaleza, CE - Brasil - 2010

\begin{tabular}{|c|c|c|c|c|c|}
\hline Autores & Ano & País & Método & Sujeitos/Local & Instrumento \\
\hline $\begin{array}{l}\text { Marin-Reyes; Rodri- } \\
\text { guez-Morán. }\end{array}$ & 2001 & México & $\begin{array}{l}\text { Estudo Caso-Controle. } \\
\text { hipertensos com } \\
\text { adesão }=\text { caso/ sem } \\
\text { adesão }=\text { controle }\end{array}$ & $\begin{array}{l}80 \text { hipertensos } \geq 35 \text { anos, } \\
\text { realizado em ambulatório } \\
\text { hospitalar. Relação caso- } \\
\text { controle 1:1. }\end{array}$ & $\begin{array}{l}\text { Questionário validado (autores } \\
\text { não especificaram o nome do } \\
\text { instrumento) }\end{array}$ \\
\hline Tuesca-Molina et al. & 2006 & Espanha & $\begin{array}{l}\text { Transversal de base } \\
\text { populacional. }\end{array}$ & $\begin{array}{l}4000 \text { adultos } \geq 60 \text { anos não } \\
\text { institucionalizados. Pesquisa } \\
\text { domiciliar. }\end{array}$ & Teste de Morisky-Green \\
\hline Afonso; Vea; Ábalo. & 2008 & Cuba & Estudo Metodológico & $\begin{array}{l}114 \text { hipertensão essencial, } \\
>20 \text { anos em tratamento anti- } \\
\text { hipertensivo. }\end{array}$ & Questionário MBG \\
\hline $\begin{array}{l}\text { Block; Melo; } \\
\text { Nogueira. }\end{array}$ & 2008 & Brasil & Coorte & $\begin{array}{l}200 \text { hipertensos resistentes de } \\
\text { um hospital universitário. }\end{array}$ & Teste de Morisky-Green \\
\hline Gohar et al. & 2008 & Reino Unido & $\begin{array}{l}\text { Inquérito descritivo } \\
\text { transversal. }\end{array}$ & $\begin{array}{l}196 \text { hipertensos (multiétinico) } \\
\text { em uso de terapia } \\
\text { complementar. Em ambulatório } \\
\text { hospitalar }\end{array}$ & $\begin{array}{l}\text { Hill-Bone compliance to high } \\
\text { blood pressure therapy scale }\end{array}$ \\
\hline $\begin{array}{l}\text { Santa-Helena; Nemes; } \\
\text { Eluf-Neto. }\end{array}$ & 2008 & Brasil & Estudo Metodológico & $\begin{array}{l}46 \text { hipertensos, Unidade } \\
\text { básica de Saúde, selecionados } \\
\text { randomicamente. }\end{array}$ & $\begin{array}{l}\text { Questionário QAM-Q, Teste } \\
\text { de Morisky-Green, Teste de } \\
\text { Haynes }\end{array}$ \\
\hline $\begin{array}{l}\text { Velandia-Arias; } \\
\text { Rivera-Alvarez }\end{array}$ & 2009 & Colômbia & Descritivo correlacional & $\begin{array}{l}201 \text { pessoas com algum fator } \\
\text { de risco cardiovascular. }\end{array}$ & $\begin{array}{l}\text { Cuestionário de valoración del } \\
\text { comportamiento de adherencia } \\
\text { al tratamiento farmacológico y } \\
\text { no farmacológico }\end{array}$ \\
\hline Dosse et al. & 2009 & Brasil & $\begin{array}{l}\text { Exploratório descritivo, } \\
\text { através de questionário } \\
\text { aplicado por telefone. }\end{array}$ & $\begin{array}{l}68 \text { hipertensos não controlados } \\
\text { de um ambulatório hospitalar } \\
\text { ( } \mathrm{PA} \geq 140 \times 90 \mathrm{mmHg})\end{array}$ & Teste de Morisky-Green \\
\hline $\begin{array}{l}\text { Mendoza-Parra; } \\
\text { Merino; Barriga. }\end{array}$ & 2009 & Chile & $\begin{array}{l}\text { Exploratório descritivo, } \\
\text { amostra randomizada }\end{array}$ & $\begin{array}{l}211 \text { hipertensos } \geq 65 \text { anos em } \\
\text { uso de fármacos e sem co- } \\
\text { morbidades, Centro de Saúde } \\
\text { da Família. }\end{array}$ & Escala de conductas en salud \\
\hline
\end{tabular}

O Quadro 1 mostra que os estudos que avaliam a adesão ao tratamento da hipertensão por meio de questionários validados vêm aumentando ao longo da década analisada, com um aumento da frequência nos anos de 2008 e 2009. Uma provável explicação para esse crescimento é o interesse dos profissionais de saúde em entender o fenômeno da adesão/ não adesão que traz grande impacto na morbimortalidade de pessoas acometidas por problemas crônicos de saúde ${ }^{(12)}$.

Percebe-se também que a problemática da avaliação da adesão ao tratamento da hipertensão arterial, conside- rando todo o ônus que essa morbidade traz para a sociedade, demanda esforços de especialistas de vários países em busca da elucidação da prevalência da adesão. Dentre os estudos selecionados, sete foram realizados no continente Americano sendo: três no Brasil, um no México, um em Cuba, um na Colômbia, um no Chile; e dois no continente Europeu: um na Espanha e um no Reino Unido. Uma revisão mostrou que vários países vêm desenvolvendo pesquisas em busca de um índice de adesão ao tratamento medicamentoso da hipertensão arterial no mundo ${ }^{(13)}$. 
Quanto ao delineamento dos estudos, há um aprofundamento no tema estudado, visto que as pesquisas mostram desenhos observacionais e analíticos procurando conhecer o território da adesão ao tratamento da hipertensão arterial e correlacioná-lo com algumas variáveis, em busca de subsídios que equacionem o problema. Desse modo, os estudos distribuíram-se em: um caso controle, uma coorte, um inquérito, dois estudos metodológicos, um transversal de base populacional, dois exploratórios-descritivos e um descritivo correlacional, segundo definições dos próprios estudos.

O delineamento de pesquisa refere-se ao plano geral do pesquisador para responder às questões de pesquisa ou suas hipóteses ${ }^{(14)}$. Nos artigos estudados, considerando as amostras randomizadas, dois estudos tinham delineamentos de pesquisa experimental.

Quanto aos sujeitos envolvidos nas pesquisas, houve variação no tamanho das amostras, o que pode ser explicado pela diversidade de locais em que as pesquisas foram realizadas e os variados delineamentos dos estudos. No entanto, oito estudos consideraram sujeitos de pesquisa pessoas com hipertensão arterial, apenas um selecionou a amostra por pessoas que apresentam algum fator de risco cardiovascular, incluído na presente revisão porque todos os sujeitos da amostra eram hipertensos. Assim, acerca dos critérios de inclusão das amostras, temos que: um estudo considerou os hipertensos descontrolados, dois, os hipertensos idosos, um, os hipertensos resistentes, e quatro, os hipertensos cadastrados em unidades de saúde de Atenção Primária.

Quanto aos Instrumentos de avaliação da adesão ao tratamento da hipertensão arterial, tem-se que a utilização de questionários validados diz respeito a instrumentos metodológicos que avaliam o comportamento do usuário para além do manejo empírico de dados que se obtém pela simples observação da conduta cotidiana ou habitual do usuário. A Tabela 1 lista os sete instrumentos levantados na presente revisão.

Tabela 1 - Uso de questionários validados para mensurar a adesão ao tratamento da hipertensão segundo os estudos selecionados - Fortaleza, CE - Brasil - 2010

\begin{tabular}{ll}
\hline $\begin{array}{l}\text { Questionário validado para mensurar adesão } \\
\text { ao tratamento }\end{array}$ & N \\
\hline Teste de Moriski e Green & 4 \\
Cuestionário de Valoración de Adherencia & 1 \\
Questionário MBG & 1 \\
Questionário QAM-Q & 1 \\
Teste de Haynes & 1 \\
Escala de conductas em salud & 1 \\
Hill-Bone compliance to high blood pressure therapy & 1 \\
scale &
\end{tabular}

\begin{tabular}{ll}
\hline Total & 10 \\
\hline
\end{tabular}

O teste de Moriski-Green foi o mais utilizado, estando presente em quatro estudos. Trata-se de uma medida construída em 1986 e constitui-se no instru- mento mais utilizado para medir adesão ao uso de medicamentos. A teoria fundamental desta medida afirma que o uso inadequado de medicamentos ocorre em uma ou em todas as seguintes formas: esquecimento, falta de cuidado, interromper o medicamento quando sentir-se melhor ou interromper o medicamento quando sentir-se pior. É de fácil medida, validado, com um número relativamente pequeno de questões compreensíveis, que proporcionam a verificação da atitude do usuário frente à tomada de medicamentos $^{(15)}$. É um instrumento qualitativo e as perguntas são atemporais ${ }^{(16)}$. Encontra-se validado em inglês, espanhol e português.

O Cuestionário de valoración del comportamiento de adherencia al tratamiento farmacológico y no farmacológico foi utilizado em um estudo(17). Trata-se de um questionário utilizado em sua versão original pelas autoras, que passou por processo de validação de conteúdo por enfermeiras experts na Colômbia tomando como referência os indicadores e a escala semântica da Taxonomia NOC (Nursing Outcomes Classification). Consta de 14 itens tipo Likert com cinco alternativas de resposta onde um, é igual a nunca significa o valor mais baixo e cinco, igual a sempre o valor mais alto. A escala encontra-se disponível em espanhol.

O desenvolvimento e validação de questionários para avaliar a adesão ao tratamento foi objeto de dois estudos ${ }^{(6,18)}$. O Questionário MBG (Martín-Bayarre-Grau), desenvolvido na Escola Nacional de Saúde Pública de Cuba, foi elaborado a partir da definição de adesão terapêutica, desenvolvida pelos autores: ação ativa e voluntária do usuário a adotar um comportamento relacionado com o cumprimento do tratamento acertado de mútuo acordo com o seu médico ${ }^{(18)}$.

O Questionário MBG é auto-aplicável, curto, de fácil aplicação e apresenta cômodas possibilidades de resposta por parte do usuário. Encontra-se validado apenas em língua espanhola, cujo processo ocorreu em Havanna, Cuba. Possui 12 itens em forma de afirmações, com resposta tipo Likert com cinco possibilidades de resposta que vão de sempre a nunca. Para calcular a pontuação obtida por cada usuário, considerou-se que o valor 0 corresponde a nunca, 1 a quase nunca, 2 às vezes, 3 quase sempre e 4 sempre, sendo 48 a totalidade dos pontos possíveis de alcançar. Os autores ainda estandarizam os valores considerando as pontuações obtidas em: aderidos totais os que obtêm 38 a 48 pontos; aderidos parciais de 18 a 37 pontos, e não aderidos de 0 a 17 pontos, de modo que se possa quantificar com rapidez e determinar três níveis de aderência: total, parcial e não aderido. O questionário apresentou uma consistência interna Alfa de Cronbach de 0,889 e a validação de conteúdo foi realizada por experts ${ }^{(18)}$.

O outro questionário desenvolvido foi o Questionário de Adesão a Medicamentos - Qualiaids (QAM-Q)
Utilização de questionários validados para mensurar a adesão ao tratamento da hipertensão arterial: uma revisão integrativa Borges JWP, Moreira TMM, Rodrigues MTP, Oliveira CJ 
elaborado para abordar o ato (se o indivíduo toma e o quanto toma de seus medicamentos), o processo (como ele toma o medicamento no período de sete dias, se pula doses, se toma de modo errático, se faz feriados) e o resultado de aderir (no caso, se sua pressão estava controlada) junto a usuários com AIDS em tratamento antirretroviral(6).

O QAM-Q possui três medidas de não-adesão: 1) Proporção de doses consumidas - medida contínua do ato de aderir: número de comprimidos consumidos multiplicado pelo número de vezes, dividido pelo número de comprimidos prescritos multiplicado pelo número de vezes; 2 ) Processo de tomadas - medida ordinal do processo de aderir: frequência de ocorrência de abandono (não tomou nenhuma dose de todos os medicamentos nos últimos sete dias), feriados (o usuário não toma qualquer medicamento naquele dia), tomada errática (deixa de tomar medicamentos em dias e horários variados), ou meia-adesão (toma corretamente um medicamento e outro de maneira incorreta); e, finalmente, 3) Desfecho referido - medida dicotômica do resultado de aderir: relato da última medida de pressão arterial, informando se estava normal ou alterada ${ }^{(6)}$.

Os pesquisadores supracitados aplicaram o QAM-Q em hipertensos e construíram uma medida composta, na qual a presença de uma dessas condições era suficiente para classificar o entrevistado como não-aderente: ou não tomar a quantidade correta ( $80 \%-120 \%$ das doses prescritas), ou não tomar de modo correto (sem feriados, tomada errática, abandono ou meia-adesão), ou relatar que sua pressão arterial estava alterada. As medidas de acurácia para detectar não-adesão mostraram sensibilidade de $62,5 \%$ e especificidade de $85,7 \%$, área sob a curva ROC de $74,1 \%$ e valor preditivo positivo de $90,9 \%$. O estudo de validação para usuários com hipertensão ocorreu em Blumenau, Santa Catarina, Brasil e é encontrado apenas em português ${ }^{(6,16)}$.

Outro instrumento para avaliar a não-adesão foi o Teste de Haynes-Sackett ${ }^{(6)}$. Nesse teste o usuário faz um auto-relato da adesão ao tratamento por meio de uma pergunta, aqueles que têm uma adesão igual ou superior a $80 \%$ são considerados aderentes? ${ }^{(19)}$.

A Escala de conductas en salud foi utilizada em um estudo no Chile ${ }^{(20)}$. Os autores utilizaram uma escala modificada para caracterizar a adesão ao tratamento. A soma das pontuações por resposta (nunca= 1 ; às vezes $=$ 2; sempre $=3$ ) permite alcançar de 12 a 36 pontos. Quatro sub-escalas indagam sobre a dieta, atividades físicas, tomada de medicamentos e se podem controlar situações de estresse em cada um dos contextos em que se desenvolve a vida diária (em casa, na recreação e nas atividades sociais). A escala original tem um formato Likert de cinco alternativas de respostas que permite alcançar um máximo de 100 pontos e conta com uma quinta sub-escala que indaga sobre o hábito de fumar e se estende ao âmbito laboral. Apesar das modificações feitas pelos autores, a escala ainda obteve pontuações aceitáveis de consistência interna alfa de Crobach entre 0,70 e 0,85 . Validado apenas em espanhol.

A escala Hill-Bone compliance to high blood pressure therapy scale (Hill-Bone compliance scale) foi utilizada em um estudo ${ }^{(21)}$, avalia comportamentos do usuário por três importantes domínios comportamentais de tratamento de pressão arterial elevada: 1) reduzir a ingestão de sódio, 2) comparecimento a consultas e 3) tomar a medicação. Esta escala é composta por 14 itens em três sub-escalas. Cada item é uma escala de quatro pontos do tipo Likert e foi desenvolvida e validada pela Escola de Enfermagem da Universidade John Hopkins, Baltmoore, EUA ${ }^{(22)}$. Escala validada em língua inglesa.

Acerca dos Escores de adesão ao tratamento da hipertensão, é difícil detectar a adesão e, mais ainda, quantificá-la. Apesar de a adesão ser frequentemente descrita como variável dicotômica adesão $x$ não-adesão, ela pode variar a um longo de um contínuo de zero a mais de $100 \%$ em usuários que usam mais do que as medicações prescritas pelo médico ${ }^{(13)}$.

A Figura 1 mostra os valores de prevalência da adesão encontrados a partir da aplicação de questionários validados. Esses escores, apesar de apresentados em conjunto, apresentam a limitação de não poderem ser comparados em uma relação linear devido primeiramente às diferenças entre os questionários, pois medem diferentes construtos relacionados à adesão e segundo as características amostrais.

Na Espanha, um grupo de pesquisadores, ao aplicarem o Teste de Morisky-Green em uma amostra de 4.000 pessoas maiores de 60 anos, encontraram uma prevalência de $53 \%$ de um bom comportamento terapêutico(23). Os autores estratificaram os resultados de acordo com o gênero e não houve diferença estatisticamente significante, tendo uma prevalência de 52,3\% de adesão em homens e 53,9 em mulheres. Em um hospital de referência do Rio de Janeiro, Brasil, foi encontrado $51 \%$ de adesão em amostra de hipertensos resistentes, utilizando o mesmo instrumento ${ }^{(24)}$. Segundo os autores o Teste de Morisky-Green não apresentou um bom desempenho nessa população, quando utilizado isoladamente, necessitando de outros métodos em associação para avaliar os hipertensos resistentes ${ }^{(24)}$.

No sul do Brasil foi encontrada uma prevalência de $56,6 \%$ de adesão com o Teste de Morisky-Green ${ }^{(6)}$. Esse resultado foi utilizado como padrão ouro combinado com outros dois métodos (adesão pelo relato do médico e adesão pelo relato do usuário), na avaliação de um questionário que estava em construção. Um dos limites desse achado é a amostra pequena $(n=46)$, o que prejudica a interpretação desse resultado. 


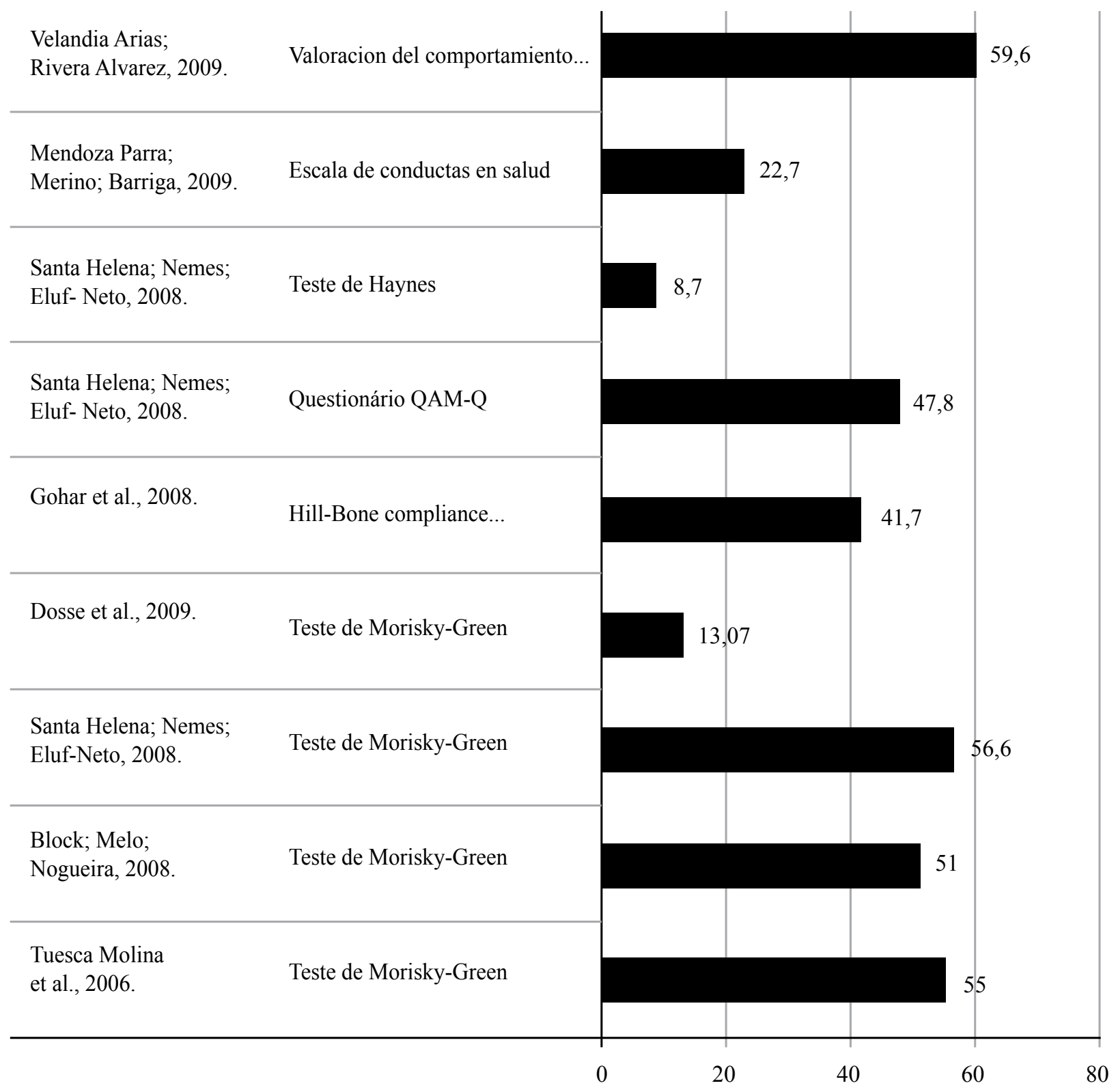

Figura 1 - Escores de adesão ao tratamento da hipertensão arterial resultantes de questionários validados, segundo os estudos selecionados - Fortaleza, CE - Brasil - 2010

Em São Paulo, Brasil, a aplicação do mesmo teste em uma amostra de 68 hipertensos de um hospital obteve uma prevalência de $13,07 \%^{(25)}$. Essa baixa adesão pode estar associada a um viés de seleção, pois a amostra foi constituída de hipertensos com descontrole pressórico, cujo critério de inclusão foi ter a pressão arterial maior que $140 \times 90 \mathrm{mmHg}$.

A utilização da Escala Hill-Bone resultou em 41,7\% de adesão em amostra de 66 hipertensos de variadas etnias em Birmingham, Reino Unido ${ }^{(21)}$. Essa escala foi utilizada em uma versão modificada para avaliar o comportamento do usuário quanto à atitude de tomada dos medicamentos. A escala original é americana e a adaptação utilizada não passou por processo de validação, o que pode comprometer a fidedignidade dessa prevalência encontrada.

Discutindo a utilização do Questionário QAM-Q, a sua aplicação resultou em uma prevalência de $47,8 \%$ de adesão no sul do Brasil. O estudo aponta algumas limitações: o pequeno número de casos, a realização em um único contexto e com apenas uma patologia, o que pode limitar a generalização dos resultados. Contudo o QAM-Q parece ser capaz de obter resultados semelhan- 
tes ou melhores quando combinado a outros métodos objetivos e subjetivos de medir adesão. Outro método utilizado no mesmo estudo, o Teste de Haynes que mede a adesão à medicação nos últimos 30 dias, a prevalência encontrada foi $8,7 \%$ de adesão(6). E essa baixa adesão pode estar associada ao viés de memória, podendo esse, influenciar negativamente o método.

No Chile, a aplicação da Escala de conductas em salud ${ }^{(20)}$ mostrou uma adesão de $22,7 \%$ em uma amostra de 211 hipertensos maiores de 64 anos de idade. 0 estudo alerta que este resultado pode ter viés devido ao estado mental dos indivíduos, ou mesmo ao uso de medicações que alteram o sistema nervoso central. Na Colômbia, a utilização do Cuestionário de valoracion del comportamiento de adherencia al tratamiento farmacológico y no farmacológico mostrou uma adesão de 59,6\% em uma amostra de 201 pessoas, os autores enfatizam que os usuários da amostra apresentam grande dificuldade para aderir ao tratamento não farmacológico(17).

\section{CONCLUSÃO}

Com esta revisão, pretendeu-se contribuir para a abordagem de um tema relevante para a saúde pública mundial e brasileira que é a mensuração da adesão ao tratamento da hipertensão. Embora existam diferentes instrumentos que podem ser usados para medir a adesão ao tratamento da hipertensão, não há um instrumento que se adeque a todos os estudos e cujos resultados possam ser comparados. Assim, são necessárias pesquisas que elaborem e validem questionários direcionados ao usuário hipertenso de forma a melhorar a qualidade de vida destes usuários e a diminuir a demanda pelos serviços de saúde.

\section{REFERÊNCIAS}

1. Prieto L, Badia X. Cuestionarios de salud: concepto y metodología. Aten Primaria. 2000;28(3):201-9.

2. Graziano KU, Padilha KG. Construction and adaptation of management and health care measures in nursing [editorial]. Rev Esc Enferm USP [Internet]. 2009 [cited 2010 Sept 12];43(n. espe): 989-9. Available from: http://www.scielo.br/scielo. php?script=sci_arttext\&pid=S0080-62342009000500001\&Ing $=$ pt\&nrm=iso\&tlng=en

3. Araújo EAC, Andrade DF, Bortolotti SLV. Item response theory. Rev Esc Enferm USP [Internet]. 2009 [cited 2010 Sept 12] ;43(n.espe):1000-8. Available from: http://www.scielo.br/ pdf/reeusp/v43nspe/en_a03v43ns.pdf

4. World Health Organization (WHO). Adherence to long term therapies: evidence for action. Geneva; 2003.

5. Souza WA. Avaliação da adesão ao tratamento e dos resultados clínicos e humanísticos na investigação da hipertensão arterial [tese doutorado]. Campinas: Universidade Estadual de Campinas; 2008.

6. Santa-Helena ET, Nemes MIB, Eluf-Neto J. Desenvolvimento e validação de questionário multidimensional para medir não-adesão ao tratamento com medicamentos. Rev Saúde Pública. 2008;42(4):764-7.

7. Gusmão JL, Ginani GF, Silva GV, Ortega KC, Mion Jr D. Adesão ao tratamento em hipertensão arterial sistólica isolada. Rev Bras Hipertens. 2009;16(1):38-43.

8. Borges JWP. Métodos de avaliação da adesão ao tratamento da hipertensão arterial: uma revisão integrativa da literatura [monografia]. Fortaleza: Universidade Estadual do Ceará; 2010.
9. Pasquali L. Psychometrics. Rev Esc Enferm USP [Internet] 2009 [cited 2010 Apr 12];43(n.esp): 992-9. Available from: http://www.scielo.br/pdf/reeusp/v43nspe/en_a02v43ns.pdf

10. Mendes KS, Silveira RCCP, Galvão MC. Revisão integrativa: método de pesquisa para a incorporação de evidências na saúde e na enfermagem. Texto Contexto Enferm. 2008;17(4):758-64.

11. Souza MT, Silva MD, Carvalho RC. Revisão integrativa: o que é e como fazer. Einstein. 2010;8(1):102-6.

12. Reiners AAO, Azevedo RCS, Vieira MA, Arruda ALG. Produção bibliográfica sobre adesão/não-adesão de pessoas ao tratamento de saúde. Ciênc Saúde Coletiva. 2008; 13 Supl 2:2299-306.

13. Barbosa RGB, Lima NKC. Índices de adesão ao tratamento anti-hipertensivo no Brasil e mundo. Rev Bras Hipertens. 2006;13(1):35-8.

14. Polit DF, Beck CT, Hungler BP. Fundamentos de pesquisa em enfermagem: métodos, avaliação e utilização. 5a ed. Porto Alegre: Artmed; 2004.

15. Ungari AQ. Adesão ao tratamento farmacológico de usuários hipertensos seguidos nos núcleos de saúde da família do município de Ribeirão Preto, SP [dissertação]. Ribeirão Preto: Faculdade de Medicina, Universidade de São Paulo; 2007.

16. Santa-Helena ET. Adesão ao tratamento farmacológico de usuários com hipertensão arterial em unidades de saúde da família em Blumenau, SC [tese doutorado]. São Paulo: Faculdade de Medicina, Universidade de São Paulo; 2007. 
17. Velandia-Arias A, Rivera-Álvarez LN. Agencia de autocuidado y adherencia al tratamiento en personas con factores de riesgo cardiovascular. Rev Saúde Pública. 2009;11(4): 538-48.

18. Alfonso LM, Vea HDB, Ábalo JAG. Validación del cuestionario MBG (Martín-Bayarre-Grau) para evaluar la adherencia terapéutica en hipertensión arterial. Rev Cub Salud Pública [Internet]. 2008 [citado 2010 set. 14];34(1). Disponível em: http://scielo.sld.cu/scielo.php?script=sci_ arttext\&pid=S0864-34662008000100012\&lng=es\&nrm=iso \&tlng=es

19. Melchiors AC. Hipertensão arterial: análise dos fatores relacionados com o controle pressórico e a qualidade de vida [dissertação]. Curitiba: Universidade Federal do Paraná; 2008.

20. Mendoza-Parra S, Merino JM, Barriga OA. Identificación de factores de predicción del incumplimiento terapéutico en adultos mayores hipertensos de una comunidad del sur de Chile. Rev Panam Salud Pública. 2009;25(2):105-12.
21. Gohar F, Greenfield SM, Beevers DG, Lip GYH, Jolly K. Self-care and adherence to medication: a survey in the hypertension outpatient clinic. BMC Complement Altern Med. 2008;8:4.

22. Kim MT, Hill MN, Bone LR, Levine DM. Development and testing of the Hill-Bone compliance to High Blood Pressure Therapy Scale. Prog Cardiovasc Nurs. 2000;15(3):90-6.

23. Tuesca-Molina R, Guallar-Castillón P, Banegas-Banegas JR, Graciani-Pérez RA. Determinantes del cumplimiento terapêutico en personas mayores de 60 años en España. Gac Sanit. 2006;20(3):220-7.

24. Bloch KV, Melo NA, Nogueira AR. Prevalência da adesão ao tratamento anti-hipertensivo em hipertensos resistentes e validação de três métodos indiretos de avaliação da adesão. Cad Saúde Pública. 2008;24(12):2979-84.

25. Dosse C, Cesarino CB, Martin JFV, Castedo MCA. Fatores associados a não adesão dos usuários ao tratamento de hipertensão arterial. Rev Latino Am Enferm. 2009;17(2):201-6. 